\title{
Philosophiques
}

\section{Jean-Guy Meunier, Genèse du matérialisme dans les écrits de jeunesse de Karl Marx, Collection « Philosophica », V. 19, Ottawa, Canada, Éditions de l’Université d'Ottawa, 1981, 131 p.}

\section{François Tournier}

Volume 13, numéro 1, printemps 1986

URI : https://id.erudit.org/iderudit/203309ar

DOI : https://doi.org/10.7202/203309ar

Aller au sommaire du numéro

Éditeur(s)

Société de philosophie du Québec

ISSN

0316-2923 (imprimé)

1492-1391 (numérique)

Découvrir la revue

Citer cet article

Tournier, F. (1986). Jean-Guy Meunier, Genèse du matérialisme dans les écrits de jeunesse de Karl Marx, Collection " Philosophica », V. 19, Ottawa, Canada,

Éditions de l'Université d'Ottawa, 1981, 131 p. Philosophiques, 13(1), 155-164.

https://doi.org/10.7202/203309ar d'utilisation que vous pouvez consulter en ligne.

https://apropos.erudit.org/fr/usagers/politique-dutilisation/ 
JEAN-GUY MEUNIER, Genèse du matérialisme dans les écrits de jeunesse de Karl Marx, Collection «Philosophica», V. 19, Ottawa, Canada, Éditions de l'Université d'Ottawa, 1981, $131 \mathrm{p}$.

\section{par François Tournier}

Paru depuis bientôt cinq ans, ce petit livre fascinant et novateur n'a pas reçu à notre avis l'attention qu'il mérite du milieu intellectuel dont il est issu. Dans le but de corriger cette situation et de souligner l'originalité de cette entreprise théorique, nous voudrions entreprendre ici une discussion qui dépasse le cadre habituel d'un simple compte rendu.

J.G. Meunier propose en fait un nouveau «mode de réflexion», une nouvelle perspective analytique et rigoureuse pour ce secteur des études marxiennes. Sur la base de cette nouvelle approche, il soulève un ensemble de questions tout à fait pertinentes et originales dont il nous faudra désormais tenir compte. En ce sens, l'A. a atteint son but. Essentiellement, Meunier nous invite à « relire » d'une façon nouvelle les écrits de jeunesse de Marx afin de reformuler plus adéquatement la thèse déjà ancienne selon laquelle ce dernier a fait ouvre «scientifique» en mettant en pratique (sans la théoriser) une nouvelle «méthode scientifique».

\section{Qu'est-ce que «dé-lire» Marx?}

L'A. appartient à un courant de pensée qui considère la lecture spontanée et intuitive des écrits de Marx comme une façon nettement insuffisante d'en comprendre la véritable «signification». À la manière de physiciens de laboratoire testant systématiquement des "hypothèses » de lecture en les confrontant aux «faits» de l'écriture, ces penseurs exigent une «re-lecture» des textes de Marx afin d'en déceler le «non-dit» au moyen d'un outillage intellectuel des plus sophistiqué. Alors qu'Althusser suggère une lecture "symptomale» fondée en partie sur le structuralisme, la psychanalyse et l'épistémologie bachelardienne, Meunier propose une lecture « logico-sémantique» s'appuyant en partie sur la grammaire générative et transformationnelle et en partie sur son épistémologie « analyticoconstructiviste». 
À vrai dire, pourquoi pas? Certes cela change les habitudes de pensée mais si nous avons accepté déjà une lecture "symptomale», sur quelle base pourrions-nous bien nous objecter à une lecture «logico-sémantique» ou même «thermodynamique» de ces écrits? Après tout, il ne s'agit que de «métaphores», c'est-à-dire de «modèles» (ou d'instruments d'analyse) empruntés à des disciplines ayant des objets différents. Nous conviendrons certainement qu'il n'est guère plus « réaliste» de considérer les écrits de Marx comme un «rêve" à déchiffrer que de soutenir qu'ils sont structurés comme un "langage» (au sens de la linguistique contemporaine). Faisons néanmoins «comme si», car l'important n'est pas le réalisme des suppositions fondamentales de ces «re-lectures» mais bien leurs implications, c'est-à-dire la qualité des nouvelles interprétations ainsi obtenues. En ce sens, on pourrait presque parler d'une aile « instrumentaliste» du marxisme.

Meunier retrace habilement la genèse du système conceptuel « profond » des écrits de jeunesse de Marx en l'organisant autour d'un noyau central appelé «matérialisme». Cependant, le matérialisme dont il est ici question ne doit plus être regardé comme une «thèse » explicite ou un «thème» de réflexion manifeste. Il s'agit plutôt d'un «mode de réflexion», c'est-à-dire une réflexion fondée sur les catégories d'un système conceptuel. L'analyse linguistique proposée entend passer des «structures de surface» (le vocabulaire, les objets, les thèmes et les problèmes explicitement formulés) aux «structures profondes» (le système permettant de "générer»- pour reprendre la métaphore de Chomsky - la signification des énoncés manifestes du discours). Ce système profond ne s'étant pas constitué d'un seul coup, Meunier en raconte le développement historique afin de mettre en évidence le champ sémantique et la grammaire textuelle qui régit le discours de surface du jeune Marx.

Le germe de cette structure conceptuelle est une attitude critique à ce point caractéristique de la pensée marxienne qu'elle ne cessera jamais d'être à l'œuvre même dans ses écrits de maturité. À ses débuts, Marx tente un peu maladroitement de définir cette attitude dans le schème conceptuel traditionnel de la matière et de la forme. Sa grande originalité consiste à dire que la matière et la forme constituent une unité indissociable. Une analyse ne peut donc s'en restreindre aux propriétés de ces deux éléments mais doit également considérer leurs relations réciproques dont la compréhension passe nécessairement par l'intermédiaire de concepts. C'est pourquoi, dans sa Dissertation doctorale de 1841, Marx préfère le système d'Épicure qui dépasse la simple «matérialité» de l'atome à laquelle se limitait Démocrite, en considérant également sa «forme» (ou son «essence»). Pour le jeune Marx, cela signifie dépasser les apparences premières afin de dégager l'essence de la réalité. Cependant, à l'arrière-plan de cette terminologie aristotélicienne et hégélienne, se tient une attitude critique face à la "substantification» des concepts. Il est clair pour Meunier que, dès cette époque, l'usage que fait Marx des termes de «matière» et de «forme» en fait des concepts non pas «métaphysiques» (synthétique a priori) mais analytiques. Comme il l'écrit (p. 34): 
"Ils sont les catégories d'un langage analytique et non les corrélats métaphysiques d'une ontologie spiritualiste. Ils définissent un schème d'analyse, un ordre de raisonnement que Marx reprendra dans de nombreux écrits, mais dans un tout autre vocabulaire et sans l'opposition formelle de la matière et de la forme.»

À l'aide de ce schème conceptuel traditionnel, le jeune Marx pose les bases d'un nouvel «instrument d'analyse» dans lequel le concept de "matière» prendra de plus en plus d'importance par rapport à celui de «forme». On ne peut pas analyser la forme d'une situation sans reconnaître qu'elle est nécessairement liée à la matière (ou à ses « conditions matérielles d'existence»).

Mais ne nous y trompons pas. La «problématique» véritable de Marx n'est pas «métaphysique» (ou ontologique) mais épistémologique et gnoséologique. En profondeur, il faut comprendre ce matérialisme naissant comme une thèse méthodologique voulant que dans toute analyse la forme soit «réduite» à la matière - Meunier parle même de son discours «réducteur». C'est pourquoi le jeune Marx reproche aux économistes classiques d'en rester uniquement à la forme des phénomènes (à des lois abstraites et à des généralisations descriptives) et de ne pas «critiquer» (c'est-à-dire «expliquer») ces apparences premières : «Expliquer n'est pas décrire.» (p. 39).

Tel est donc le discours «critique» du jeune Marx: il consiste maintenant à «réduire» les formes apparentes (comme la monnaie, l'argent, le profit, le capital, etc.) à leur «matérialité», c'est-à-dire aux relations réciproques entre les différents éléments composant le système social dont ces formes ne sont que l'expression la plus manifeste. Au cours de cette seconde période (Manuscrits de 1844 et Idéologie allemande), le système conceptuel marxien d'origine se modifie considérablement. Non seulement Marx abandonne-t-il la dualité matière et forme avec le vocabulaire qui lui est lié, mais il redéfinit son «matérialisme» comme une théorie des rapports. Ce nouveau concept de « rapport social» s'inscrit au coeur d'un autre système conceptuel et ne peut en conséquence être compris que par rapport à un ensemble d'autres concepts qui lui sont sémantiquement liés.

Malgré ces profondes transformations, la problématique du jeune Marx demeure foncièrement épistémologique. En effet, il ne refuse pas tant les objets, le vocabulaire, les thèmes et les problèmes des économistes classiques que leur façon d'aborder les phénomènes économiques. Ceux-ci considèrent les notions économiques purement descriptives qu'ils ont élaborées comme des réalités objectives alors que ce ne sont que des concepts nous permettant de faire apparaître des « rapports» déterminant les agents sociaux en apparence «libres». Smith et Ricardo sont donc coupables de «substantification» de concepts.

Ainsi se constitue progressivement un réseau conceptuel « profond» se manifestant à la «surface» par un renouveau du vocabulaire de Marx. En 
surface, le matérialisme marxien devient une théorie des conditions matérielles d'existence, c'est-à-dire une théorie des rapports et des modes de production.

"Cette proposition, écrit Meunier (p. 68), de l'autonomie des rapports de production et leur détermination dans une forme quelconque nous apparaît une des caractéristiques les plus fondamentales du matérialisme du jeune Marx.»

Ce second réseau conceptuel subira à nouveau des transformations importantes dans les écrits subséquents de Marx - l'histoire logicosémantique de ses écrits de maturité reste encore à écrire. L'important pour Meunier (et pour nous qui ignorions cet aspect implicite de sa pensée) est que le jeune Marx utilise son système conceptuel comme s'il s'agissait d'un simple «instrument d'analyse» (un langage) permettant de «critiquer» (expliquer) les apparences premières.

\section{Profits et pertes}

Puisque nous ne pouvons «évaluer» ces «re-lectures» que par la « richesse» des interprétations qu'elles permettent de formuler, considérons à présent ce que nous gagnons $(G)$ et ce que nous perdons $(P)$ avec celle proposée par Meunier.

$\left(G_{1}\right)$ : Il ne fait aucun doute que son entreprise théorique réhabilite les écrits de jeunesse de Marx qui, depuis l'intervention d'Althusser, ont été largement dévalorisés. Une comparaison avec la façon dont les historiens des sciences ont systématiquement dévalorisé la science médiévale avant le $\mathrm{XX}^{\mathbf{e}}$ siècle n'est pas sans rapport ici. C'est certainement un "gain» majeur que de dénoncer de tels "préjugés» qui consistent à croire que l'œuvre de maturité de Marx et la science moderne se sont constituées sur le mode d'une "génération spontanée» ou d'une « conversion».

$\left(G_{2}\right)$ : En proposant sa lecture logico-sémantique des écrits du jeune Marx, l'A. fait apparaître le caractère largement «métaphorique» de la lecture «symptomale» et, de ce fait, le caractère « instrumentaliste» de l'épistémologie althussérienne. En ce domaine, Althusser fait exactement ce qu'il reprochait à Jacques Monod de faire en ce qui a trait à la biologie et à l'étude des sociétés humaines.

«Le processus de pensée et son organisation conceptuelle en discours théorique ne sont compris ici qu'à travers les concepts physique de "coupure" et psychanalytique de "symptôme".

(p. 20)

Or, il n'est pas du tout évident que ce soient là les métaphores les plus appropriées pour parler du discours de Marx. En effet, c'est sur le modèle de l'analyse d'un "rêve» (ou d'un "symptôme») qu'Althusser fait ressortir le «non-dit» des textes de Marx ou des 
jeux de mots de Lénine. En ce sens, comme le suggère avec justesse Marx, des instruments logico-sémantiques paraissent beaucoup plus appropriés pour parler de la "structure interne» d'un discours - il ne s'agit tout de même pas d'analyser sa «personnalité».

$\left(\mathrm{G}_{3}\right)$ : En considérant les écrits de Marx comme un «langage» (au sens linguistique et «non-marxiste» du terme) avec ses aspects syntaxiques, sémantiques et pragmatiques (donc comme des énoncés de surface régis par une grammaire textuelle profonde), Meunier est à même de «démontrer» formellement que certaines interprétations "classiques» de ces écrits, sont incompatibles avec son réseau conceptuel profond et, en conséquence, ne peuvent appartenir au champ sémantique de ses écrits de jeunesse. Ainsi sont disqualifiées les interprétations suivantes du matérialisme de Marx :

$\left(I_{1}\right)$ : la «matière» (au sens de la physique contemporaine) est le fondement explicateur de la réalité ;

$\left(\mathrm{I}_{2}\right)$ : la «matière» (au sens de la métaphysique et de l'ontologie traditionnelles) est la "cause première» de toute chose l'interprétation en termes de « Hegel renversé» ;

$\left(\mathrm{I}_{3}\right)$ : le matérialisme est une attitude «positiviste» devant la connaissance (la description rigoureuse de la réalité objective en termes de « lois», construites et acquises à partir de l'expérience et de la vérification);

$\left(\mathrm{I}_{4}\right)$ : le matérialisme est un «humanisme» (en parlant d'«aliénation», le jeune Marx proposerait un discours éthique eft philosophique);

$\left(I_{5}\right)$ : le matérialisme du jeune Marx n'est que le prolongement du matérialisme de Feuerbach - selon Meunier (p. 41), "c'est avant tout ce (son) mode critique de pensée que Marx retiendra».

$\left(G_{4}\right)$ : L'entreprise théorique de Meunier permet enfın de «démontrer» formellement et d'une façon beaucoup plus élégante que ne l'avait fait Althusser avant lui, que le «matérialisme» de Marx est une «épistémologie», une conception de l'étude « scientifique » des faits sociaux. Tout au long de ses écrits de jeunesse, Marx se serait conformé à cette «méthodologie» implicite qui, selon Meunier, consiste à ne pas «substantifier» des concepts. L'application d'un modèle linguistique conduisant à mettre en évidence le «nominalisme» du jeune Marx est certainement l'apport le plus original et intéressant de la recherche menée par Meunier.

Considérons les pertes à présent. Ce qu'on gagne d'un côté, on le perd évidemment de l'autre. Ainsi sont «perdues» : 
$\left(\mathrm{P}_{1}\right)$ : les interprétations « classiques» $\left(\mathrm{I}_{1}\right)$ à $\left(\mathrm{I}_{5}\right)$ du matérialisme de Marx que Meunier vient de disqualifier;

$\left(\mathrm{P}_{2}\right)$ : l'épistémologie althussérienne qui est remplacée par l'épistémologie « analytico-constructiviste» de Meunier;

$\left(\mathrm{P}_{3}\right)$ : la méthode de lecture «symptomale» et la catégorisation du développement intellectuel de Marx qu'elle implique.

$\left(\mathrm{P}_{4}\right)$ : Enfin, si les occasions ont gagné un partisan, les « réalistes» viennent d'en «perdre» un. L'interprétation de Meunier conduit en effet à reléguer à l'arrière-plan le « réalisme» (une autre interprétation "classique») du discours de surface en opérant une scission entre son «instrument d'analyse» (syntaxe et sémantique) et ses actions et ses buts explicites (pragmatique).

On imagine facilement la réplique « réaliste». En s'engageant concrètement dans des luttes idéologiques et politiques (la «surface » des écrits de Marx, devons-nous dire), il est certain que ce dernier voulait dépasser le simple plan «épistémologique» et «scientifique». En travaillant, par exemple, à la création d'un parti communiste international, il n'avait certainement pas l'impression d'élaborer («en profondeur») un «système conceptuel». Si les «classes sociales», le «prolétariat», l'«exploitation», l'«aliénation», etc., ne sont que des concepts, c'est nécessairement les "substantifier» que de prétendre les «transformer» concrètement par l'action politique. Pour préconiser un changement dans les « rapports sociaux» par une révolution politique, ne faut-il pas postuler à un endroit ou à un autre de la chaîne de notre raisonnement que cette expression fait référence à une réalité objective autre qu'à une simple façon de se représenter la réalité ? En fait, il ne s'agit pas tant, pour Marx, de décrire ou même d'«expliquer» scientifiquement ces rapports sociaux. Marx ne voulait pas tant «transformer» des «systèmes conceptuels» que la réalité économique et sociale de son temps. D'ailleurs, dans ses écrits, son petit ton sarcastique n'est-il pas là pour nous rappeler qu'il est à l'extrême opposé de l'attitude neutre, objective et entièrement rationnelle du physicien de laboratoire. Marx a clairement un «parti pris».

Faisons les comptes. Formellement, il y aura « profit», c'est-à-dire que l'interprétation de Meunier $\left(\mathrm{I}^{\mathrm{M}}\right.$ ) est « acceptable si et seulement si » (accpt ssi),

$$
I^{M} \text { accpt ssi } G_{n}^{M}-P_{n}^{M}>O,
$$

où « $G_{n}^{M}$ 》 représente les gains $\left(G_{1}\right.$ à $\left.G_{4}\right)$ de son interprétation et « $P_{n}^{M}$ » représente ses pertes $\left(\mathrm{P}_{1}\right.$ à $\left.\mathrm{P}_{4}\right)$. En d'autres termes, si les gains et les pertes de l'interprétation de Meunier s'équivalent ( $\leq 0$ ), alors il ne reste que la beauté de la «métaphore». On pourrait «adoucir» ce critère d'acceptabilité absolu avec un critère d'acceptabilité relatif comme le suivant :

$$
I^{M} \text { accpt ssi } G_{n}^{M}-P_{n}^{M}>G_{k}^{A}-P_{k}^{A}: I^{A}
$$


où « $I^{A}$ » représente l'interprétation d'Althusser, « $G_{k}^{A}$ » et « $P{ }_{k}^{A}$ » les gains et les pertes de cette interprétation. En se fondant sur de tels critères d'acceptabilité, on pourrait décider « rationnellement» d'adopter la « métaphore» de Meunier plutôt que celle d'Althusser.

Mais nous touchons évidemment ici aux limites des « démonstrations 》 formelles. En effet, pour donner une signification au symbole « $>$ » dans les formules (1) et (2), il faudrait déterminer un ordre de grandeur pré-établi et comparer la «valeur» des variables substituées aux constantes dans ces formules. Cependant, pour accorder une «valeur» plus grande au «nominalisme» de $\operatorname{Marx}\left(\mathrm{G}_{4}\right)$ qu'à son «réalisme» $\left(\mathrm{P}_{4}\right)$, il ne faut pas tant une «échelle de grandeur» qu'une "échelle de valeur». L'impasse est d'autant plus. infranchissable qu'on peut considérer la première échelle comme un sous-ensemble de la seconde: en effet, la préférence pour les «démonstrations» formelles et l'utilisation de «modèles» abstraits présupposent une telle «échelle de valeur».

\section{La «scientification» de Marx}

Si Meunier conteste la méthode de lecture et l'interprétation d'Althusser, ce n'est pas sans reprendre, par ailleurs, le projet fondamental de son école de pensée: celui de «scientificiser» le discours de Marx non pas en y apportant des améliorations théoriques mais en lui apposant une «étiquette » contrôlée (si on peut dire). En effet, l'A. prétend montrer «comment, dans ses premiers écrits, le fonctionnement intellectuel de Marx et la production de ses idées instaurent déjà dans le domaine du discours scientifique, une pratique théorique» (p. 101), voire même «une pratique nouvelle de la science» (p. 117).

Nous pourrions surnommer cette nouvelle méthodologie de la science le «nominalisme critique» si ce n'est que l'A. préfère les qualificatifs d' «analytique» et de «constructiviste». Dans cette approche «formalisante», il semble inconcevable de vouloir évaluer la «scientificité» du discours de Marx avec des «règles» de construction autres que les siennes propres. Ainsi, il est inapproprié de confronter ce discours à des critères épistémologiques comme ceux de Russell, des néo-positivistes contemporains ou de Popper. Marx tout jeune s'est opposé (pour ne pas dire qu'il a "dépassé») cette forme d' «empirisme naï » qui conduit à de simples généralisations descriptives sans explication véritable. Le danger est de «substantifier» des concepts et de s'engager ainsi dans des confusions de « niveaux» de langage. Meunier (note 19, p. 106) croit même avoir repéré une telle confusion dans le modèle $\mathrm{D}-\mathrm{N}$ de l'explication scientifique élaboré par la tradition anglo-saxonne. À son avis, on y identifie l' «explicitation» (la déduction réglementée à partir d'une prémisse) à l'«explication» ( l'affirmation de la vérité d'une prémisse»). Cependant, à notre avis, Meunier n'est pas justifié d'imputer une telle confusion à cette tradition de recherche qui distingue très nettement l'«explication» (expression anglaise) de l'«explanation» (expression anglaise). 
Dès ses écrits de jeunesse, Marx aurait donc mis en pratique cette épistémologie «formalisante» qui consiste à élaborer des «systèmes conceptuels» (des «instruments d'analyse») permettant d'« expliquer» (en dépassant les «apparences») la réalité tout en évitant de les substantifier. On ne peut évidemment espérer « vérifier» ces systèmes à partir de «ce que l'expérience immédiate "positive" pourrait en saisir» (p. 112) - comme le suggèrent les «empiristes naïfs ». Pour Marx, comme pour Meunier semble$\mathrm{t}$-il, la science ne doit pas se limiter à décrire abstraitement des « prétendus faits» mais à démontrer les liens, les rapports et les relations qui les unissent: "le véritable lieu du discours scientifique qui est analyse de rapports.» (p. 112). C'est donc une telle épistémologie « analytico-constructiviste» et "pragmatique» (parce qu'au service de l'action) qui serait déjà en "germe» dans les écrits de jeunesse de Marx. Ce n'est pas peu dire car, de continuer Meunier (p. 113):

«Le formalisme, comme discours de la science n'est pas une question de vogue, mais une règle constitutrice du discours scientifique contemporain.»

Meunier et les althussériens ont certainement tort de ranger aussi rapidement les néo-positivistes et les popperiens sous le même vocable d' «empirisme naïf»: d'une part, parce que ces deux courants de pensée soutiennent des thèses incompatibles sur ces questions et, d'autre part, parce que ces épistémologues ne donnent pas la même signification que Meunier au mot «empirisme» et ne sont donc pas aussi «naïfs» qu'on voudrait bien le faire croire. Force nous est de reconnaître que Popper ne partage pas non seulement l'argumentation «anti-empiriste» de Meunier mais également ce que nous pourrions appeler son "échelle de valeurs» :

«En cet âge postrationaliste que nous vivons, un nombre d'ouvrages toujours plus grand sont écrits dans des langages symboliques et il est de plus en plus difficile de voir pourquoi : quel est, en définitive, leur propos et pourquoi il devrait être nécessaire ou avantageux de consentir à être assommé de volumes constitués de banalités présentées sous une forme symbolique. On dirait presque que le symbolisme devient une valeur en soi, qu'il faut vénérer pour sa sublime «exactitude». Il s'agit là d'une nouvelle expression de la vieille recherche de la certitude, un nouveau rituel symbolique, un nouveau substitut de la religion.»

(La logique de la découverte scientifique, Paris, Payot, 1973, p. 401)

Popper n'hésiterait pas, semble-t-il, à recourir au mot «idéologie» pour faire référence à cette nouvelle «religion de l'exactitude». Non pas qu'il s'oppose aux définitions rigoureuses et aux formulations précises mais il est très au fait de ce que certains avant lui ont surnommé le «scientisme en science sociale». L'apparence la plus immédiate des discours scientifiques respectés d'aujourd'hui étant une utilisation «massive» de formalisme et de modèles abstraits, il est tentant de les imiter superficiellement, c'est-à-dire 
en copiant simplement cet aspect, dans les sciences sociales. Des transpositions un peu trop rapides d'instrument d'analyse d'un secteur de la recherche à un autre (sans tenir compte de la spécificité de leur objet respectif) n'est pas sans inquièter ce grand maître de l'épistémologie contemporaine. En bref, l'épistémologie «analytico-constructiviste» de Meunier est tout à fait dans le ton de cette «idéologie» de la science dont Popper voit croître la popularité non sans entretenir certaines craintes.

La difficulté que nous voudrions soulever est la suivante : en omettant, comme Meunier, d'indiquer clairement les limites d'une analyse «logicosémantique» et en imputant cette « idéologie» contemporaine de la science au jeune Marx, ne commettons-nous pas tout simplement un anachronisme - tout en profondeur soit-il ? Il est généralement reconnu que Marx a très certainement cherché à faire œuvre "scientifique» mais cela ne pouvait être que conformément à l'« idéologie» de la science de son époque et non à celle qui prévaudra cent ans plus tard. Sans nécessairement les assimiler à des choses aussi éphémères que des changements de «mode», il faut néanmoins reconnaître que les conceptions de la science (les «épistémologies») changent comme les théories scientifiques. L'«idéologie» de la science du $\mathrm{XIX}^{\mathrm{e}}$ siècle était frappée sur le modèle de la mécanique newtonienne. Or, s'il est une chose qui distingue les physiques newtonienne et einsteinienne (surtout depuis son interprétation dite de Copenhague), c'est bien le « réalisme» de leurs «suppositions».

Marx vivait à une époque de «révolutions sociales» importantes où l'on pouvait presque observer les « luttes de classes», c'est-à-dire des masses d'individus (des «forces sociales») s'affrontant dans des guerres civiles. De là à penser que le résultat de l'interaction de ces «forces sociales» donne lieu à ce que nous appelons l' " histoire humaine» et que cette histoire pouvait s'expliquer un peu à la manière dont Newton expliquait la trajectoire d'une planète par la combinaison des actions d'une force d'inertie et d'une force d'attraction, il n'y avait qu'un pas à franchir. Découvrir les lois de combinaison des «forces sociales» devint donc son « rêve» scientifique. Cependant, ce que Marx emprunta ainsi à la science de son temps, ce n'est pas le «formalisme» de la théorie newtonienne mais bien son idée principale, c'est-à-dire le « réalisme» de ses suppositions. Bien au contraire, ce sont ses contradicteurs « marginalistes » qui reprendront le calcul intégral et différentiel et proposeront, un peu plus tard, une épistémologie «instrumentaliste » assez similaire à celle, « analytico-constructiviste», de Meunier.

Cependant, ces propos ne sont pas pour contredire la thèse centrale de l'A. selon laquelle le «matérialisme» du jeune Marx est une nouvelle façon «critique» (sans substantifier les concepts) de comprendre les phénomènes sociaux. C'est un acquis majeur de son analyse que de montrer la présence d'une forme de "nominalisme» dans les écrits de jeunesse de Marx. La méthode de lecture de Meunier apporte un éclairage nouveau sur ces questions et met ainsi en évidence un aspect jusque-là ignoré de sa pensée. Mais, à trop vouloir insister sur cet aspect, nous risquons de reléguer à 
l'arrière-plan l'aspect historique («pragmatique» pour reprendre l'expression de Meunier) de ces écrits. Il y a chez le jeune Marx non seulement une philosophie implicite de la pratique théorique (une épistémologie) mais également une "philosophie sociale» explicite.

L'importance à accorder à chacun de ces aspects semble complètement déterminée, chez Meunier, par le « rapport» (purement linguistique) entre des structures de «surface» et des structures "profondes». N'est-ce pas là cependant, prendre la «métaphore» linguistique un peu trop au sérieux - voire même la substantifier? A vouloir expliquer, comme le grammairien générativiste, les structures de surface (l'explicite dans les écrits de Marx) par des structures profondes, ne risquons-nous pas de perdre à nouveau ce qui nous semblait un acquis important de sa recherche, à savoir de réhabiliter les écrits de jeunesse de Marx? Encore une fois, comme dans le cas de la lecture althussérienne, les écrits de Marx sont "dévalorisés». En effet, dans les deux cas, ces écrits ne sont pas pris au sérieux, pour ce qu'ils sont, c'est-à-dire des appels à l'équité sociale - peu importe le «vocabulaire» et les «catégories» utilisées. Afin de ne pas devoir renier les apports souvent intéressants et novateurs de ces « re-lectures» des écrits de Marx, il importe, pensons-nous, de bien spécifier les limites des « métaphores» que l'on impose ainsi aux textes.

Département de philosophie

Université du Québec à Montréal 